\title{
Reflexiones de los Aportes de la Declaración de Salamanca 25 Años Después
}

\section{Reflections on the Contributions of the Salamanca Statement 25 Years Later}

\author{
Catalina Andújar Scheker * \\ OEI, República Dominicana
}

\begin{abstract}
Este artículo tiene como objetivo reflexionar sobre los aportes de la Declaración y Marco de Acción de Salamanca a la educación inclusiva y cómo el apoyo se constituye en el factor clave para responder a las dificultades que por diversas causas experimentan los estudiantes en la escuela y posibilitar así que tengan éxito en sus aprendizajes. Para estos fines, se analiza brevemente el contexto en que ocurre la Conferencia de Salamanca y su contribución a una educación más justa, equitativa e inclusiva, tratando los avances y desafíos que existen hoy día para su concreción en el contexto escolar. Se abordan de manera sucinta las condiciones necesarias para escuelas inclusivas poniendo un especial énfasis en el apoyo a los aprendizajes, que aparece como un denominador común de los mejores sistemas educativos del mundo. A manera de ejemplo, se presentan algunas experiencias que se han desarrollado en República Dominicana en las últimas décadas relacionadas con el apoyo a los aprendizajes y que posteriormente han sido asumidas como políticas públicas. Finalmente, se reflexiona en torno a los riesgos de una visión limitada y segmentada en torno al tema hasta tratar cómo está siendo concebida y abordada la educación inclusiva hoy día, en el marco de los Objetivos de Desarrollo Sostenible y la Agenda 2030 de Naciones Unidas. Por último, se destaca la necesidad de que los países adopten políticas para impulsar marcos integrales de apoyo en sus sistemas educativos, para posibilitar así el aprendizaje de todos los niños, sin ningún tipo de exclusión.
\end{abstract}

Descriptores: Educación para todos; Aprendizaje; Apoyo pedagógico; Oportunidades educativas; Calidad de la educación.

This article aims to reflect on the contributions of the Salamanca Statement and Framework for Action to inclusive education and how learning support is the key factor to respond to learning difficulties that students experience for several reasons, and thus enabling everyone to have success in their learning. For these purposes, the context in which the Salamanca Conference takes place and its contribution to a more just, equitable and inclusive education are briefly analyzed, dealing with the advances and challenges that exist today in the school context. The conditions necessary for inclusive schools are briefly presented with a special emphasis on learning support, which appears as common denominator of the best education systems in the world. As an example, some experiences that have been developed in the Dominican Republic in recent decades related to learning support and that have subsequently been assumed as public policies are presented. Finally, we reflect on the risks of a limited and segmented vision around the issue until we treat how inclusive education is being considerate today, within the framework of the Sustainable Development Goals and the 2030 Agenda of United Nations. Finally, we present the need for countries to adopt policies to promote comprehensive support frameworks in their education systems, in order to enable the learning of all children, without any exclusion.

Keywords: Education for all; Learning; Tutoring; Educational opportunities; Educational quality.

*Contacto: candujar@oeidominicana.org

ISSN: 0718-7378

www.rinace.net/rlei/
Recibido: $\quad 18 / 08 / 2019$

$1^{a}$ Evaluación: 30/08/2019

Aceptado: 12/09/2019 


\section{La Declaración de Salamanca: Un camino recorrido entre luces y sombras}

La Declaración de Salamanca constituyó un hito en el debate sobre la educación para todos. No solo reafirmó los compromisos de la Conferencia Mundial celebrada en Jomtien, Tailandia, en el año 1990, sino que removió concepciones y posturas sobre el significado real de su postulado, poniendo el foco en la igualdad de oportunidades y en la equidad educativa.

En el momento histórico en que se formuló, cuatro años después de la Conferencia de Jomtien, la educación estaba pasando por procesos de cuestionamientos internos y experimentando cambios sustantivos a nivel mundial. La región latinoamericana no era ajena a esos procesos y los países estaban inmersos en reformas educativas profundas. El escenario educativo era efervescente, generándose una mayor conciencia pública sobre la necesidad e importancia de una educación calificada "para todos" (Rivero, 2000, p. 107).

La Declaración de Salamanca puso en el debate la atención a la diversidad de estudiantes que confluye en la escuela e instó a mirar a aquellos que presentaban dificultades de aprendizaje por diversas causas incluyendo condiciones de discapacidad, que tal como plantea Echeita (2006), constituyen "el grupo de alumnos más proclive a ser objeto de exclusión educativa" (p. 43). Además, suscitó el principio de la educación inclusiva creando un movimiento imprescindible para cuestionar las políticas y prácticas excluyentes (Saleh, 2004) y realizando aportes sustantivos a su concreción en el contexto escolar, a través de su Marco de Acción, al incluir lo relativo a la organización escolar, la gestión institucional, la formación docente, los recursos de apoyo, la participación de la familia y la comunidad, entre otros.

El Foro Mundial sobre la Educación realizado en Dakar (año 2000), reconoce en su Marco de Acción los compromisos educativos suscritos en la década de los 90, entre éstos los de la Conferencia Mundial sobre Necesidades Educativas Especiales de Salamanca, planteando la necesidad de actuar en base a los mismos (p.8). A partir de esa fecha, "el movimiento de la inclusión cobra más fuerza para hacer frente a los altos índices de exclusión y discriminación y a las desigualdades educativas presentes en la mayoría de los sistemas educativos del mundo" (Blanco, 2006, p. 5).

Pasando balance a los años transcurridos desde la Declaración de Salamanca y su Marco de Acción, se puede afirmar que ha habido importantes avances hacia una educación más inclusiva. La comunidad internacional la asume como un derecho y en todo el mundo se están llevando a cabo intentos de ofrecer respuestas educativas más efectivas a todos los niños, independientemente de sus características o condiciones personales o sociales (Ainscow y Echeita, 2010, p. 3).

En la región latinoamericana, la educación inclusiva forma parte de la agenda de los gobernantes, está incorporada en políticas, planes nacionales, leyes y normativas, plasmada en los planes de estudios de los sistemas educativos e incluida en programas de formación del profesorado; además es objeto de campañas públicas, debates, programas y proyectos (Andújar, 2014).

Sin embargo, aún persisten importantes desafíos para hacer efectivo el derecho de todos a una educación inclusiva de calidad (Blanco, 2008). Si bien es cierto que el acceso a la educación se ha incrementado significativamente en los países de la región, y que se accede 
desde etapas más tempranas (BID, 2012), al 2008 de aproximadamente 117 millones de niños, niñas y adolescentes que hay en América Latina y el Caribe en edad de asistir a la educación inicial, primaria y secundaria básica, 6.5 millones no asisten a las escuelas y 15.6 millones asisten a éstas arrastrando una historia repetitiva de fracasos y señales de desigualdad (UNICEF, UIS, 2015, p. 17), hasta terminar fuera del ámbito educativo.

En su mayor parte, son niños, niñas y adolescentes con discapacidad, provenientes de pueblos originarios, afrodescendientes, inmigrantes, que viven en condiciones de pobreza y en zonas rurales apartadas. Esta población difícilmente encuentra oportunidades educativas de calidad para poder transitar por los diferentes niveles educativos, acceder a ofertas educativas flexibles e incorporarse al mercado productivo.

La Declaración de Salamanca y el Marco de Acción para las Necesidades Educativas Especiales, movilizó y sensibilizó a gobernantes, tomadores de decisiones, académicos, agencias cooperantes, familias y sociedades en torno a una educación más justa y equitativa. A través de sus principios impulsó un cambio de enfoque basado en la superación de las barreras que todos encuentran dentro del sistema educativo (Ainscow, 2004). Pero, su concreción en el contexto escolar ha sido un proceso complejo plagado de dificultades. Más de dos décadas después de su aprobación, es imperativo reflexionar y analizar sobre cómo sus principios y pautas han sido traducidos por los sistemas educativos y materializados en las escuelas, a fin de identificar las oportunidades para superar los múltiples desafíos que persisten.

\section{Llegando a todos los estudiantes y a cada uno de ellos: Aportes para una educación inclusiva}

Uno de los rasgos distintivos de la Declaración de Salamanca fue haber puesto el foco en el niño, en su individualidad, en el sentido de la educación. Murillo y Krichesky (2015) afirman que el centro de una escuela que mejora son los alumnos, no las prácticas de enseñanza, ni la gestión, que sólo cobran sentido en función de éstos. Para estos autores esto significa "aceptar radicalmente a los alumnos de cada centro tal como son y adaptar a ellos el proceso de enseñanza-aprendizaje y no a la inversa" (p. 76).

La Educación Inclusiva se basa en las transformaciones que debe hacer todo centro educativo para posibilitar que todo estudiante, independientemente de sus características individuales y de los contextos con los que interactúa, participe de la experiencia educativa y mejore sus aprendizajes. La inclusión pone particular énfasis en aquellos grupos de alumnos que podrían estar en riesgo de marginalización, exclusión o fracaso escolar (Ainscow y Echeita, 2011).

El Marco de Acción de Salamanca trazó directrices para orientar políticas, planes y acciones, señalando diversos factores que son necesarios para dar una respuesta efectiva a la diversidad en el contexto escolar y que, por tanto, están presentes en una educación de calidad. Estos elementos, vinculados al contexto escolar, se definen en función de las necesidades de aprendizaje de los estudiantes. A continuación, abordaremos brevemente algunos de ellos y las pautas que se plantean:

- El currículo: la necesidad de que los planes de estudio sean flexibles y respondan a todos los estudiantes es una condición necesaria para las escuelas inclusivas. En este sentido, el currículo se configura como un proceso facilitador u 
obstaculizador del aprendizaje y el rendimiento escolar de todos los estudiantes. Coll y Martin (2006) llaman la atención sobre el riesgo de la sobrecarga de contenidos o competencias en el currículo escolar para el aprendizaje significativo y funcional, planteando además que suponen "una dificultad añadida para seguir avanzando hacia una educación inclusiva” (p. 10).

- La evaluación: debe asumirse como un proceso continuo que permita identificar las necesidades de apoyo de los estudiantes, para orientar el trabajo del docente en el aula (Andújar y Rosoli, 2014). Se trata de una evaluación formativa, vinculada al ajuste de la enseñanza (Coll y Onrubia, 2002) a la identificación de necesidades y a la puesta en marcha de apoyos.

- El trabajo docente: el Marco de Acción de Salamanca plantea la necesidad de revisar y transformar los procesos formativos de los docentes, en sus diversos tramos, para responder a las necesidades de todos sus estudiantes. Plantea además el papel decisivo que tienen los docentes en los procesos educativos y en la prestación de los apoyos necesarios, a través del uso de diversos recursos y del trabajo colaborativo.

- La gestión institucional: El foco de la gestión institucional que se privilegia es contar con directores líderes, que promuevan el trabajo en equipo y la vinculación con la familia y la comunidad, que velen por los aprendizajes de todos sus estudiantes y sean garantes de la calidad y equidad de los procesos educativos de sus centros. En el abordaje de este elemento, el referido Marco de Acción plantea que es función del director promover el trabajo colaborativo en su centro y asumir el compromiso del éxito de cada estudiante.

- Los recursos y servicios de apoyo: el Marco de Acción de Salamanca destina diversos apartados al tema del apoyo y lo aborda como un elemento para responder a las necesidades de aprendizaje de los estudiantes. Se destaca que el apoyo debe ser un proceso continuo, que va desde una ayuda mínima hasta la aplicación de programas de apoyo pedagógico suplementarios en la escuela, ampliándolos si es necesario para recibir apoyo de personal especializado externo.

Todos estos factores han sido ampliamente abordados por diversos autores, así como por responsables de diseñar y ejecutar políticas públicas en materia educativa, constituyéndose la respuesta educativa a la diversidad en una preocupación constante para los sistemas educativos y las escuelas.

El Marco de Acción sobre Necesidades Educativas Especiales (1994) plantea cambios en la forma de concebir y abordar cada uno de estos factores, que son los mismos que se requieren para "mejorar la calidad y pertinencia de la educación, así como para la promoción de un mejor aprovechamiento escolar por parte de todos los alumnos" (p. 21). Sin negar la relevancia de cada uno de ellos, nos centraremos en el apoyo como factor esencial para una educación inclusiva y equitativa, partiendo de la necesidad inaplazable de los sistemas educativos de posibilitar oportunidades educativas de calidad para que todos los estudiantes aprendan. 


\section{El apoyo a los aprendizajes: Factor clave para una educación inclusiva y una constante de los mejores sistemas educativos del mundo}

El Marco de Acción de Salamanca plantea entre sus principios que debe darse a todos los niños la misma educación, con la ayuda adicional necesaria para aquellos que la requieran (UNESCO, 1994).

Este planteamiento, que nos remite a la concepción de apoyo pedagógico, es abordado por diversos autores desde perspectivas distintas.

Booth y Ainscow (2002) definen el concepto de "apoyo" o "apoyo pedagógico", como "todas las actividades que aumentan la capacidad de un centro educativo para atender a la diversidad del alumnado (p. 21). Para Onrubia (2009), la mejor manera de alcanzar los objetivos educativos es asegurar un amplio abanico de oportunidades educativas o apoyos para aprender, que pueda utilizarse de manera flexible, favoreciendo que distintos estudiantes o un mismo estudiante ante diversos aprendizajes o en diversos momentos de su aprendizaje puedan recibir ayudas distintas y más adecuadas. Por otro lado, Reimers (2002), aborda el tema de la igualdad de oportunidades educativas, planteando que esta existe cuando cualquier persona accede a la institución educativa y recibe el apoyo adecuado para "aprender en profundidad a niveles de excelencia y proceder al siguiente nivel educativo" (p. 151).

Andújar y Rosoli (2014), se refieren al apoyo en un centro educativo a partir de una mirada amplia de toda la escuela y no solo en función de los estudiantes que presentan mayores dificultades en sus aprendizajes. En este sentido, Blanco (2008) dice que el apoyo abarca todos aquellos recursos que complementan o refuerzan la acción pedagógica de los docentes. De acuerdo con Echeita y colaboradores (2012), el apoyo escolar en el marco de una educación inclusiva pasa a entenderse como un "conjunto de políticas, planes y acciones educativas que contribuyen, de modo convergente y sostenido en el tiempo, a mejorar la capacidad de un centro escolar para promover la presencia, el rendimiento escolar y la plena participación de todo su alumnado en condiciones de calidad" (p.10). En las últimas décadas, diversos organismos internacionales (OCDE, UNESCO, OEI, BM, BID, UNICEF), entidades y autores han vuelto la mirada al tema del apoyo y su impacto en la calidad y equidad de los sistemas educativos.

Barber (2009) en su trabajo sobre "El Desafío de lograr un Rendimiento Escolar de Nivel Mundial: La Educación en el siglo XXI", plantea que un alto desempeño supone que todos los alumnos tengan éxito. A partir de las conclusiones del informe de McKinsey \& Co de 2007 titulado "Cómo hicieron los sistemas educativos con mejor desempeño del mundo para alcanzar sus objetivos", hace referencia a un elemento común de estos sistemas educativos y es que, además de conseguir a las personas más aptas para ejercer la docencia, desarrollarlas hasta convertirlas en instructores eficientes, y tener docentes cualificados y con las competencias necesarias, todos ponen en práctica mecanismos de apoyo para garantizar que todos los niños puedan aprender. De acuerdo con el informe, se evidencia que en estos sistemas existe una preocupación por las causas del retraso educativo de los estudiantes y desarrollan estrategias para superarlo.

Barber y Mourshed (2008), analizan como estos sistemas educativos con mejor desempeño comienzan "por fijar expectativas claras y altas respecto de lo que cada alumno debe saber, 
comprender y ser capaz de hacer y luego monitorean su desempeño en comparación con las expectativas, interviniendo allí donde estas últimas no son satisfechas" (p. 39). En estos sistemas las propias escuelas implementan procesos de seguimiento e intervención para identificar a los alumnos que necesitan apoyo y brindarles ese apoyo en forma continua. En este sentido, Barber y Mourshed (2008) afirman que "los sistemas de excelencia intervienen a nivel de cada alumno, y desarrollan dentro de las escuelas procesos y estructuras capaces de identificar cuándo un estudiante está comenzando a retrasarse, interviniendo para mejorar el desempeño del niño" (p. 38).

Schleicher (2018), haciendo referencia a una gama de estudios comparativos liderados por Tucker del Centro Nacional de Educación y Economía de los Estados Unidos en torno a los sistemas de educación con un rendimiento alto evaluados por PISA, señala como características comunes a todos ellos, que "tienen altas expectativas de sus estudiantes y entienden que cualquier estudiante puede aprender, abordando la diversidad de necesidades de los estudiantes con estrategias pedagógicas diferenciadas" (p. 38). De acuerdo a los estudios referidos, estos sistemas educativos de alto rendimiento no solo reconocen la necesidad de establecer políticas y acciones que aseguren la formación de docentes de calidad, sino que también orientan a los docentes para impulsar estrategias de apoyo en el aula para que los estudiantes tengan éxito en sus aprendizajes (Schleider, 2018).

La OCDE (2016) plantea que un programa para promover aprendizajes de calidad en todos los estudiantes debe estar vinculado a "superar las barreras existentes, crear un ambiente en los centros escolares en el que se apoye a los alumnos, ofrecer medidas de apoyo suplementario lo antes posible, promover la participación de padres y comunidades locales y motivar a los alumnos a que saquen el máximo partido de las oportunidades educativas disponibles" (p. 7). Estos planteamientos de la OCDE están en consonancia con los principios y directrices establecidos en la Declaración y Marco de Acción de Salamanca en el año 1994, que posteriormente fueron abordados en el Foro Mundial de Educación para Todos de Dakar en el año 2000 y en la Conferencia Mundial de Incheon del año 2015.

Para hacer efectivo el derecho a una educación inclusiva de calidad es necesario garantizar que todos los estudiantes aprendan, prestando especial atención a aquellos que más ayudas necesitan (Blanco, 2008). El apoyo se constituye asi en un factor imprescindible para avanzar, siempre y cuando los sistemas educativos lo asuman desde una perspectiva sistémica e integral y generen las condiciones para que los centros educativos puedan poner en marcha sus propias modalidades y estrategias de apoyo, a partir de las necesidades identificadas.

\section{Algunas experiencias de apoyo a los aprendizajes en República Dominicana: De proyectos a políticas públicas}

\subsection{Centros de recursos para la atención a la diversidad}

En el año 2003, desde la Dirección de Educación Especial del Ministerio de Educación de República Dominicana se puso en marcha un proyecto para la creación de una red nacional de Centros de Recursos para la Atención a la Diversidad en República Dominicana, con apoyo de la Agencia Española de cooperación Internacional para el Desarrollo (AECID). 
El proyecto respondía a las directrices del Marco de Acción de Salamanca y su objetivo era brindar apoyo a los centros educativos para responder a las necesidades educativas especiales de sus estudiantes, así como a sus familias. La propuesta inicial abarcó la creación de dos centros, que se adecuaron y equiparon para tales fines. Se contrató a un equipo de profesionales externos, que se capacitó, para intervenir en el contexto escolar, acompañando a los equipos docentes en las evaluaciones y proponiendo estrategias para dar respuestas a las necesidades educativas específicas identificadas. Los centros además apoyaban la labor docente y desde allí se suministraban materiales educativos y otros recursos para dar apoyo a los niños con necesidades educativas especiales y sus familias.

Esta experiencia focalizada en dos centros fue ampliada a trece centros de recursos, en todo el territorio nacional, quedando instaurada en el sistema educativo dominicano. Aunque esta red de centros se convierte en una oportunidad para fortalecer los apoyos y llegar a más estudiantes que lo requieren, su marco de actuación deberá revisarse a la luz de los avances en torno a la educación inclusiva, para evitar intervenciones externas y descontextualizadas, que no tomen en cuenta los procesos educativos escolares que podrían estar ocasionando, manteniendo o intensificando las dificultades de aprendizaje.

\subsection{Aulas de recursos para el aprendizaje}

La estrategia de Aula de Recursos para el Aprendizaje fue diseñada por la Organización de los Estados Iberoamericanos para la Educación, la Ciencia y la Cultura (OEI) y puesta en marcha en la República Dominicana a partir del año 2009 en 12 escuelas de un distrito educativo en la región este del país, en el marco del proyecto de Mejora de la Calidad Educativa, implementado como parte de una amplia estrategia de colaboración con los países iberoamericanos para diseñar políticas e impulsar prácticas que promuevan la mejora de la calidad y de la equidad educativa. Su objetivo era brindar apoyo pedagógico a estudiantes de los primeros grados que presentaban dificultades de lectura y escritura con el fin de mejorar sus aprendizajes.

El Aula de Recursos para el Aprendizaje (ARPA) funcionaba en un espacio equipado con diversos recursos educativos, materiales y tecnológicos, utilizados para el trabajo con pequeños grupos de estudiantes y para la realización de proyectos innovadores vinculados con los procesos de alfabetización inicial. Los apoyos pedagógicos se proporcionaban de manera transitoria, en contra turno a grupos pequeños de estudiantes que lo requerían, a partir de la realización de una evaluación diagnóstica llevada a cabo por el docente. Los grupos de estudiantes estaban integrados además por estudiantes que presentaban buenos desempeños en esas áreas, que fungían como tutores. Como responsables de las aulas, se contaba con facilitadoras.

Una lección aprendida de esta experiencia es la necesidad de involucrar a las familias, para una mayor participación de sus hijos y la necesidad de un mayor involucramiento del docente de aula. Además, se requiere una mayor articulación entre este espacio y el aula. Faltó potenciar el aula como espacio de apoyo, con actividades de aprendizaje cooperativo que fortalecieran los aprendizajes de todos los niños.

Esta experiencia fue la base del Sistema Integral de Apoyo a los Aprendizajes, definido en el año 2011 y asumido posteriormente por el Ministerio de Educación de República Dominicana como un componente de la Política de Apoyo a los Aprendizajes de lectura, escritura y matemática en los primeros grados del nivel básico. Su estructura fue acogida por el Ministerio de Educación con los espacios de apoyo a los aprendizajes, que gestiona 
la Dirección de Educación Especial del Ministerio de Educación. aunque difiere en su enfoque conceptual y en los mecanismos que regulan su forma de operar.

4.3. El sistema integral de apoyo a los aprendizajes en los centros educativos: Una experiencia llevada a cabo en la región este de República Dominicana

El Sistema Integral de Apoyo a los Aprendizajes es un ámbito de actuación definido por OEI en el año 2011, en el marco de la implementación de la Política del Ministerio de Educación de República Dominicana de Apoyo a los Aprendizajes de lectura, escritura y matemática en los primeros grados.

El objetivo es atender el fracaso escolar y evitar que los estudiantes se queden rezagados, con el fin último de que los estudiantes aprendan. Este Sistema se define desde una mirada amplia de toda la escuela y no solo en función de los niños y las niñas que presentan mayores necesidades en sus aprendizajes. Abarcó además la reorientación de los recursos del centro educativo, y la elaboración y uso de recursos distintos que sirvieron para complementar la labor docente.

El Sistema Integral de Apoyo a los Aprendizajes se implementó en 86 centros educativos en tres regiones educativas de la República Dominicana. A cada centro educativo se les dieron orientaciones, formándose a los equipos directivos y docentes, para que pudiesen definir su propio sistema integral de apoyo, a partir de los recursos existentes en su centro y las necesidades identificadas. El sistema que se implementó priorizó las estrategias de trabajo colaborativo como mecanismo de apoyo no solo pedagógico, sino también al trabajo docente y directivo. Entre estas estrategias se destacan: las duplas docentes, las redes de directores y las redes docentes.

Para dar respuesta a las dificultades para el aprendizaje de la lectura, la escritura y la matemática, se estableció la estrategia de apoyo directo en espacios habilitados para estos fines (Arpas). Estos apoyos nunca se daban individuales y siempre ocurrían a contraturno, es decir en un horario distinto que el horario clase. El Sistema Integral de Apoyo a los Aprendizajes descrito ha quedado plasmado en algunos documentos institucionales y se han impulsado algunas de sus acciones en iniciativas puntuales que se han llevado a cabo, pero en la práctica no ha sido acogido de manera formal por las instancias educativas.

Las experiencias anteriormente referidas han aportado al debate en torno a los apoyos pedagógicos para favorecer los aprendizajes de los estudiantes, de manera específica a aquellos estudiantes que se van quedando atrás. Su adopción como políticas públicas en expansión afirma la necesidad de impulsar desde la propia estructura, un sistema de apoyo que responda a las dificultades de aprendizaje que presentan los estudiantes por diversas causas y donde confluyan una serie de estrategias que partan del aula y centro y que impliquen a todos los involucrados a través de redes de colaboración.

\section{Mirando al futuro: Reafirmando los principios de la Declaración de Salamanca y transformando las políticas y prácticas para que todos los estudiantes aprendan}

La Declaración de Salamanca hizo suyo el concepto de inclusión (Ainscow y Miles, 2008, p. 18) poniéndolo en la agenda educativa y social de los países. Abrió además el debate sobre el significado de una única educación para todos, incluyendo a los más excluidos, 
que parecía no estar siendo visibilizado en la Declaración de Educación para Todos de Jomtien (Ainscow, 2008), referida más bien a una educación "para la mayoría” (Blanco, 2008). Sin embargo, estos innegables aportes podrían estar siendo amenazados por subjetividades. En algunos países parece difícil desligar el concepto de educación inclusiva del de educación especial del criterio de necesidades educativas especiales, o más recientemente del término de necesidades educativas de apoyo específico, llevándose a cabo en la práctica acciones puntuales para atender "lo diverso", lo que pone en riesgo la concepción misma de inclusión. Hoy día, a partir de los compromisos establecidos por los países para el cumplimiento del Objetivo de Desarrollo Sostenible 4 de "garantizar una educación inclusiva, equitativa y de calidad y promover oportunidades de aprendizaje permanente para todos" al 2030, esta visión parece limitada. Este objetivo mundial obliga a los sistemas educativos a transformarse y repensar sus estructuras para que la búsqueda de mejores resultados en los aprendizajes esté acompañada de oportunidades educativas de calidad para todos.

En este marco, el apoyo a los aprendizajes debe formar parte de un sistema integral que involucre a todos los que participan de la experiencia educativa, que se lleve a cabo de manera continua y que esté disponible no solo para el estudiante particular, sino además para los demás alumnos y los equipos docentes. Sin duda el mayor reconocimiento de los aportes de la Declaración de Salamanca y su Marco de Acción es que 25 años después de su formulación, la educación inclusiva aparece como núcleo central del Objetivo de Desarrollo Sostenible centrado en la educación (OEI, 2018), y sus principios permean los compromisos de Inclusión y Equidad de la Agenda 2030 de "hacer frente a todas las formas de exclusión y marginación y en centrar los esfuerzos en los más desfavorecidos, especialmente aquellos con discapacidad, para velar por que nadie se quede atrás" (UNESCO, 2015, p. 2)

\section{Referencias}

Ainscow, M. (2004). Salamanca diez años después. ¿Qué impacto ha tenido en el ámbito internacional? Recuperado de https://www.educacionespecial.sep.gob.mx/pdf/doctos/3Internacionales/5DiezSalamanc a.pdf

Ainscow, M. y Miles, S. (2008). Making education for all inclusive: Where next? Prospects, 38(1) 15-34. https://doi.org/10.1007/s $11125-008-9055-0$

Ainscow, M. y Dyson, A. Promoviendo la equidad en educación. Manchester: Centre for Equity in Education.

Ainscow, M. y Echeita, G. (2010). La educación inclusiva como derecho. Marco de referencia y pautas de acción para el desarrollo de una revolución pendiente. Recuperado de https://pdfs.semanticscholar.org/91e3/9b49fccccc56d6ad1coeoe2ed9addc9b8d6b.pdf

Andújar, C. (noviembre, 2014). Las TIC: Oportunidades, Barreras y Retos para la Educación Inclusiva. Conferencia presentada en el Congreso Iberoamericano de Ciencia, Tecnología, Innovación y Educación Avanzando juntos hacia las Metas Educativas Iberoamericanas 2021. OEI, Buenos Aires.

Andújar, J. y Rosoli, L. (2014). Enseñar y aprender en la diversidad: el desarrollo de centros y aulas inclusivos Recuperado de https://oei.org.do/Oei/Noticia/avances-y-desafios-de-laeducacion-inclusiva-en-iberoamerica 
Barber, M. (2009). El desafio de lograr un rendimiento escolar a nivel mundial. Recuperado de www.educacionfch.cl/secciones/directorio-de-recursos/recursos-gesti\%C3\%B3n-yliderazgo. html?pid=124\&sid=171:Conferencia-de-Michael-Barber

Barber, M. y Mourshed, M. (2008). Cómo hicieron los sistemas educativos con mejor desempeño en el mundo para alcanzar sus objetivos. Serie Santiago de Chile: PREAL.

Blanco, R. (marzo, 2008). Marco conceptual sobre educación inclusiva, en una breve mirada a los temas de educación inclusiva: Aportes a las discusiones de los talleres. Comunicación presentada en la Conferencia Internacional de Educación. OIE, UNESCO.

Blanco G. R.(2006). La equidad y la inclusión social: Uno de los desafíos de la educación y la escuela hoy. REICE. Revista Iberoamericana sobre Calidad, Eficacia y Cambio en Educación, 4(3), 1-15.

Booth, T., y Ainscow, M. (2002). The index for inclusion. Bristol: Centre for Studies on Inclusive Education.

Booth, T. y Ainscow, M. (2004) Índice de inclusión: Desarrollando el aprendizaje y la participación en las escuelas. Santiago de Chile: Orealc/Unesco.

Booth, T. (2011). Guía para la Educación Inclusiva: Desarrollando el aprendizaje y la participación en los centros escolares. Bristol: Centre for Studies in Inclusive Education (CSIE).

BID. (2012). Educación para la transformación. Washington, DC: Banco Interamericano de Desarrollo.

Coll, C. y Martín, E. (2006) Vigencia del debate curricular. Aprendizajes básicos, competencias y estándares. Revista PRELAC, 3, 6-23.

Coll, C. y Onrubia, J. (2002). Evaluar en una escuela para todos. Cuadernos de Pedagogía, 318, 5054.

Echeita, G. (2006). Educación para la inclusión o educación sin exclusiones. Madrid: Narcea.

Echeita, G. (2008). Inclusión y exclusión educativa. Voz y quebranto. REICE. Revista Iberoamericana sobre Calidad, Eficacia y Cambio en Educación, 6(2), 9-18.

Echeita, G. y Verdugo, M. (2004). La Declaración de Salamanca sobre necesidades educativas especiales diez años después. Valoración y prospectiva. Recuperado de https://sid.usal.es/idocs/F8/FDO9045/declaracion_salamanca_completo.pdf

Echeita, G., Sandoval, M., Simon, C. y Monarca, C. (2012). Cómo fomentar las redes naturales de apoyo en el marco de una escuela inclusiva. Recuperado de https://www.researchgate.net/publication/264287752_Como_fomentar_las_redes_natur ales_de_apoyo_en_el_marco_de_una_escuela_inclusiva_Propuestas_practicas

Murillo, F. J. y Krichesky, G. J. (2015) Mejora de la escuela: Medio siglo de lecciones aprendidas REICE. Revista Iberoamericana sobre Calidad, Eficacia y Cambio en Educación, 13(1), 69-102.

OCDE. (2016). PISA Estudiantes de bajo rendimiento. Por qué se quedan atrás y cómo ayudarles a tener éxito. Resultados Principales. Recuperado de

http://www.oecd.org/pisa/keyfindings/PISA-2012-Estudiantes-de-bajo-rendimiento.pdf

Onrubia, J. (2009). Transformar para adaptar, adaptar para incluir: una mirada psicoeducativa a la educación inclusiva. En C. Giné (Coord.), La educación inclusiva. De la exclusión a la plena participación de todo el alumnado (pp. 49-62). Barcelona: Horsori

Reimers, F. (2002). Tres paradojas educativas en América Latina. Sobre la necesidad de ideas públicas para impulsar las oportunidades educativas. Revista iberoamericana de Educación, 29, 131-155. 
Rivero, J. (2000). Reforma y desigualdad en America Latina. Equidad en la educación? Revista Iberoamericana de Educación, 23, 103-133.

Schleider, A. (2018) Primera clase. Cómo construir una escuela de calidad para el siglo XXI. Madrid: Fundación Santillana. https://doi.org/10.1787/9788468050126-es

UNESCO. (1994). Declaración de Salamanca y marco de acción para las necesidades educativas especiales. Madrid: Ministerio de Educación y Ciencia de España.

UNESCO. (2000). Marco de Acción de Dakar: Educación para Todos: cumplir nuestros compromisos comunes (con los seis marcos de acción regionales). Recuperado de https://unesdoc.unesco.org/ark:/48223/pfooo0121147_spa

UNESCO. (2015). Declaración de Incheon: Educación 2030: Hacia una educación inclusiva y equitativa de calidad y un aprendizaje a lo largo de la vida para todos. Recuperado de https://unesdoc.unesco.org/ark:/48223/pfo0o0233137_spa

UNICEF, UIS (2015): Completar la escuela un derecho para crecer, un deber para compartir. Madrid: Santillana.

\section{Breve CV de la autora}

\section{Catalina Andújar Scheker}

Licenciada en Psicología Escolar y especialista en Intervención Psicológica en Contextos Educativos por la Universidad Complutense de Madrid. Ha sido directora de Educación Especial del Ministerio de Educación de República Dominicana de 1996 a 2008. Se desempeñó como coordinadora nacional de programas educativos de la Cooperación Española y de la UNESCO, impulsando programas vinculados con la mejora de la calidad educativa, la educación inclusiva y la educación permanente de personas jóvenes y adultas. Presidenta de la Fundación Tonucci: Educando para la Diversidad del año 2004 al 2008. Fue consultora del PNUD para la reforma educativa dominicana. Ha sido docente en diversas universidades dominicanas. Autora y coautora de publicaciones vinculadas al ámbito educativo. ORCID ID: https://orcid.org/0000-0002-0663-9142. Email :

catalina.andujar@gmail.com 